\title{
BOUNDS ON THE DIMENSION OF VARIATIONS OF HODGE STRUCTURE
}

\author{
BY
}

JAMES A. CARLSON ${ }^{1}$

\begin{abstract}
We derive upper bounds on the dimension of a variation of Hodge structure of weight two and show that these bounds are sharp. Using them we exhibit maximal geometric variations of Hodge structure. Analogous results for higher weight are obtained in the presence of a nondegeneracy hypothesis, and variations coming from hypersurfaces are shown to be nondegenerate. Maximal geometric variations of higher weight are also constructed.
\end{abstract}

1. Introduction. Let $D$ be a classifying space for Hodge structures on a fixed integral lattice and let $\Gamma \backslash D$ be the associated space of isomorphism classes. If $X / S$ is a smooth family of projective algebraic varieties, then there is a natural holomorphic map $v: S \rightarrow \Gamma \backslash D$ defined by sending $s$ in $S$ to the class of the primitive cohomology group $H^{k}\left(X_{s}\right)_{0}$ in $\Gamma \backslash D$. In [11] Grothendieck considered the problem of characterizing the geometric locus of $\Gamma \backslash D$ : the subset $\mathbf{G}$ of Hodge structures which arise from geometry, that is, which are image points under some period mapping $v$. A fundamental restriction on the position of the image of $v$ is that imposed by Griffiths' transversality [10]: if $F^{*}(t)$ is a one-parameter family of Hodge filtrations deduced from $X / S$, then

$$
d F^{p} / d t \subset F^{p-1}
$$

holds. Because of this relation, the image of $v$ must be, special cases excluded, a proper analytic subvariety of $\Gamma \backslash D$. In addition, since the Hilbert scheme of varieties of fixed degree and dimension in $\mathbf{P}^{n}$ has finitely many components, the geometric locus of $\Gamma \backslash D$ must at worst be a countable union of proper subvarieties.

The purpose of this note is to exhibit components of the geometric locus. This we do by deriving bounds on the dimension of infinitesimal variations of Hodge structures $[4,5,14]$, hence, a fortiori, on variations of Hidge structures themselves. It then suffices to construct examples which satisfy the bounds.

The starting point is Griffiths' transversality: if $T(D)$ is the holomorphic tangent bundle of $D$, then the relation (GT) defines a natural subbundle $T_{h}(D)$ relative to which any variation of Hodge structure must be tangent, so that

$$
\operatorname{dim}(v(S)) \leqslant \operatorname{dim}_{f} T_{h}(D) \leqslant \operatorname{dim}_{f} T(D),
$$

Received by the editors November 21, 1984.

1980 Mathematics Subject Classification. Primary 14C30.

'Partially supported by NSF grant MCS 8102745 .

1986 American Mathematical Society $0002-9947 / 86 \$ 1.00+\$ .25$ per page 
where the subscript $f$ refers to the fiber dimension. In special cases the two bundles coincide so that $\mathbf{G}$ is potentially equal to $\Gamma \backslash D$. (Indeed $\mathbf{G}=\Gamma \backslash D$ for the period spaces of abelian varieties and of $K 3$ surfaces.) In the weight two case the fiber dimensions are

$$
\operatorname{dim}_{f} T_{h}(D)=h^{2.0} h^{1.1}
$$

and

$$
\operatorname{dim}_{f} T(D)=\operatorname{dim}_{f} T_{h}(D)+\frac{1}{2} h^{2.0}\left(h^{2.0}-1\right),
$$

so that the transversality restriction, nontrivial whenever $h^{2.0}>1$, is

$$
\operatorname{dim}(v(S)) \leqslant h^{2.0} h^{1.1} .
$$

We show that additional conditions hold.

THEOREM 1.1. Let $v: S \rightarrow \Gamma \backslash D$ be a variation of Hodge structure of weight two with $h^{2.0}>1$. Then

$$
\operatorname{dim}(v(S)) \leqslant \frac{1}{2} h^{2,0} h^{1,1} .
$$

The proof relies on the integrability conditions satisfied by variations of Hodge structure, first recognized by Griffiths and used here in the form of the commutativity axiom for infinitesimal variations [4, p. 54 (iv); 5, p. 130; 14, p. 423]. With this estimate in hand it is possible to exhibit components of the geometric locus.

EXAmPLE 1.2. Consider the elliptic surface $E$ defined by

$$
y^{2}=4 x^{3}-g_{2}(t) x-g_{3}(t),
$$

where $g_{2}$ and $g_{3}$ are polynomials of degrees 12 and 18. Let $S$ be the space of pairs of polynomials for which $E$ is smooth, and let $E / S$ be the surface over $S$ defined by $(*)$. Then $E / S$ is an elliptic surface of geometric genus 2 with two independent relative algebraic cycles, namely the classes of a section and of a fiber. Define the primitive cohomology of $E_{s}(s \in S)$ to be the orthogonal complement of the subspace spanned by these two cycles, and define a period map $v$ by sending $s$ to the primitive second cohomology of $E_{s}$. The space of infinitesimal deformations, $H^{1}\left(E_{s}, \Theta\right)$, has dimension 28 , and because local Torelli holds [12, p. 57], the image $v(S)$ in $\Gamma \backslash D$ has dimension 28 as well. On the other hand, the estimate of Theorem 1.1 becomes

$$
\operatorname{dim} v(S) \leqslant h^{1.1}=28,
$$

so that $v(S)$ is a component of $\mathbf{G}$, as desired.

Example 1.3. Let $Q$ be the quadric cone in $\mathbf{P}^{3}$ and let $Q^{\prime}$ be the natural desingularization. Let $C$ be a smooth curve in $Q$ cut out by a quintic threefold which does not pass through the vertex, let $C^{\prime}$ be its preimage in $Q^{\prime}$, and let $E$ be the exceptional locus for the blowup. Then $C^{\prime}+E$ is even in the Picard group of $Q^{\prime}$ and so there exists a double cover $X$ of $Q^{\prime}$ with $C^{\prime}+E$ as branch curve. Considerations similar to those of the preceding example show that the image of a versal family of such surfaces is a component of $\mathbf{G}$ in $\Gamma \backslash D$. Here $p_{g}=2$ and $K^{2}=1$, so the surfaces in question are general type,

An example shows that the bound of Theorem 1.1 cannot be improved as a universal, as opposed to contingent assertion about weight two variations, even for those with quasiprojective base. 
Construction 1.4: Unitary variations. Let $B$ be the generalized unit ball, defined as the set

$$
\left\{Z=p \times q \text { complex matrix }\left.\right|^{t} \bar{Z} Z<1\right\} .
$$

Let $D$ be a classifying space for polarized Hodge structures of weight two with Hodge numbers $h^{2,0}=2 p$ and $h^{1.1}=2 q$. These spaces, $B$ and $D$, are homogeneous for unitary and orthogonal groups $U(p, q)$ and $S O(2 p, 2 q)$. As we show in $\S 6$ there is an equivariant, holomorphic, and horizontal map $v: B \rightarrow D$. Since $B$ has dimension $p q$ and since $v$ is an imbedding, this unitary variation of Hodge structures realizes the bound of the theorem.

REMARK 1.5. A discrete subgroup of the unitary group acts both on $B$ and on $D$, so that one may form quotient variations

$$
v: \Gamma \backslash B \rightarrow \Gamma \backslash D
$$

with $\Gamma \backslash B$ quasiprojective. Therefore the bound is sharp in the subcategory of variations of Hodge structure with global geometric base.

As we show in Theorem 6.3, all variations of Hodge structure which satisfy the bound of Theorem 1.1 with equality are locally unitary, provided that $h^{2,0}>2$. This raises a number of questions and problems.

(P1) Do the $U(p, q)$-homogeneous variations come from geometry? Although the base $\Gamma \backslash B$ is a geometric object, one requires more: that $v$ come from the cohomology of family of varieties over $\Gamma \backslash B .^{2}$

(P2) Given a specific variation of Hodge structure, how does one decide whether it is maximal in the class of variations? If it has maximal dimension, then it is certainly maximal, but the converse is not true, as one may show by example. ${ }^{3}$

(P3) Exhibit components of the geometric locus with $h^{2,0}>2$.

The method of proof which we give for Theorem 1.1 also allows us to give bounds on variations of higher weight, subject to additional nondegeneracy hypotheses (ND1 and ND2). Although it is not entirely clear at this time to what extent these conditions are reasonable, they are neither entirely artificial nor useless, as the following indicate.

(1) All hypersurface variations satisfy ND1, and generic hypersurface variations satisfy ND2. Similar remarks should apply to broad classes of complete intersections in weighted projective spaces, in projective bundles over projective spaces which arise from a sum of line bundles, etc.

(2) The nondegeneracy conditions are open conditions on infinitesimal variations (IVHS). In addition, the conditions are stable: if $V$ is a nondegenerate IVHS and if $V^{\prime}$ is a second IVHS containing $V$, then $V^{\prime}$ is also nondegenerate.

(3) Using the dimension estimates which we give subject to ND1 and ND2, we are able to exhibit components of the geometric locus in higher weight. The image of moduli in periods for hypersurfaces of dimension $n$ and degree $n+2$ is such an

\footnotetext{
${ }^{2}$ An affirmative answer to (P1) is given in Shimura varieties of weight two Hodge structures, by James Carlson and Carlos Simpson, to appear in the Barcelona Conference on Hodge Theory (Springer-Verlag).

${ }^{3} \mathrm{~A}$ partial answer to $(\mathrm{P} 2)$ is given in Hypersurface Variations are Maximal by James Carlson and Ron Donagi, to appear. The results therein also settle (P3).
} 
example. Only for the case of quartic surfaces does this class of examples give $\mathbf{G}=\Gamma \backslash D$.

The author wishes to thank Arnaud Beauville, Herb Clemens, Mark Green, Chris Peters, Edoardo Sernesi, and Domingo Toledo, all of whose comments have contributed to the present form of this work. Special thanks are due Harsh Pittie for his careful and critical reading of an earlier version of this paper.

2. Definitions and conventions. An infinitesimal variation of Hodge structue, $V(H)$, is an object which formalizes the structure inherent in the differential of the period mapping at a single point $[4,5,14]$. To describe it, consider a polarized Hodge structure $H=\left(H_{\mathrm{z}}, F^{*},\langle\rangle,\right)$ and let $G^{\circ}(H)=\oplus G^{p}=\oplus F^{p} / F^{p-1}$ be the graded object associated to the Hodge filtration. Because of the Riemann-Hodge bilinear relations, the polarizing form $\langle$,$\rangle descends to G^{\circ}(H)$. Let End ${ }^{\circ}(H)$ be the graded ring of endomorphisms of $G^{\circ}(H)$, endowed with a Lie algebra structure in the obvious way; $[\phi, \psi]=\psi \circ \psi-\psi \circ \phi$. Let $E^{\circ}(H)$ be the subalgebra consisting of endomorphisms satisfying the condition

$$
\langle\phi(x), y\rangle+\langle x, \phi(y)\rangle=0 .
$$

(antisymmetry)

Then an infinitesimal variation of $H$ is a map

$$
\delta: T \rightarrow E \cdot(H)
$$

satisfying

$$
\delta(T) \subset E^{-1}(H)
$$

(horizontality)

and

$$
[T, T]=0 .
$$

(commutativity)

To give $V(H)$ is therefore to give the pair $(H, \delta)=\left(H, \delta: T \rightarrow E^{-1}(H)\right)$.

We shall often abuse notation and identify $T$ with its image, so that an infinitesimal variation $V(H)$ may be viewed as an abelian subalgebra of $E^{\cdot}(H)$ which is horizontal, i.e., of pure degree -1 .

The dimension of $V(H)$ is the dimension of $\delta(T)$. If $V$ and $V^{\prime}$ are infinitesimal variations of $H$ and $T$ contains $T^{\prime}$, then $V$ contains $V^{\prime}$. If $V$ is contained in no other infinitesimal variation of $H$, then $V$ is maximal. The centralizer of $V(H)$ is the subspace

$$
\mathscr{Z}(T)=\left\{\phi \in E^{-1}(H) \mid[\phi, \psi]=0 \text { for all } \psi \in \delta(T)\right\} .
$$

An infinitesimal variation is maximal if and only if it is self-centralizing.

The highest Hodge level is the integer

$$
r=\max \left\{p \mid H^{p, q} \neq 0\right\} .
$$

The lowest Hodge level is the complementary index $s$, so that $r+s=n$, where $n$ is the weight. The level $(l)$ is the number of steps in the Hodge filtration, i.e., $r-s$. A Hodge structure whose level is less than its weight should be thought of as arising from a Hodge structure of lower weight by tensor product with an appropriate tensor power of the Hodge structure of Tate: the rigid Hodge structure of weight -2 and type $(-1,-1)$ with lattice $2 \pi i \mathbf{Z} \subset \mathbf{C}$. 
The subspace of $G^{p}$ generated by $V$ is

$$
T\left(G^{p+1}\right)=\operatorname{span}\left\{\phi(x) \mid \phi \in T, x \in G^{p}\right\} .
$$

An infinitesimal variation is nondegenerate if both of the following hold:

(ND1) $T\left(G^{p}\right)=G^{p-1}$ for all $p$ for which $G^{p} \neq 0$;

(ND2) if $a=r-l-2$, then $\operatorname{dim} T^{(a)}(S) \geqslant \operatorname{dim} S$ for all subspaces $S$ of $G^{r-1}$.

Here $T^{(k)}(S)=\operatorname{span}\left\{\phi_{1} \circ \cdots \circ \phi_{\mathrm{k}}(\mathrm{x}) \mid x \in S, \phi_{i} \in T\right\}$. The indices are chosen so that $S$ lies in level (highest -1$)$ and so that $T^{(a)}(S)$ lies in the complementary level, $($ lowest +1$)$.

3. Bounds for weight two. We shall now prove Theorem 1.1 by constructing a filtration of $T$ with special properties. To this end let $w_{1}, \ldots, w_{n}$ be a basis of $G^{2}$ and set

$$
T^{k}=\left\{\phi \in T \mid \phi\left(w_{i}\right)=0 \text { for } i \leqslant k\right\},
$$

with $T^{0}=T$. The resulting sequence of annihilators defines a decreasing filtration $T^{0} \supset T^{1} \supset T^{2} \supset \cdots$ of $T$. Evaluation of $T^{k}$ on $w^{k+1}$ defines an exact sequence

$$
0 \rightarrow T^{k+1} \rightarrow T^{k} \rightarrow T^{k}\left(w_{k+1}\right) \rightarrow 0,
$$

where $T^{k}\left(w_{k+1}\right)$ is a subspace of $G^{1}$. Set $S_{k+1}=T^{k}\left(w_{k+1}\right)$ and observe that there result isomorphisms

$$
T \cong \bigoplus_{k} T^{k} / T^{k+1}, \quad T^{k} / T^{k+1} \cong S_{k+1}, \quad T \cong \bigoplus_{k} S_{k} .
$$

The proof of Theorem 1.1 will follow from a study of the $S_{k}$.

LEMma 3.1. The subspaces $S_{k}$ constructed above are mutually orthogonal.

Proof. Consider arbitrary elements $x$ and $y$ of $S_{i}, S_{j}$, where $i<j$. Then we have

$$
\begin{aligned}
\langle x, y\rangle & \left.=\left\langle\phi\left(w_{i+1}\right), \psi\left(w_{j+1}\right)\right\rangle \quad \text { (for some } \phi \text { in } T^{i}, \psi \text { in } \mathrm{T}^{\mathrm{j}}\right) \\
& =-\left\langle\psi \circ \phi\left(w_{i+1}\right), w_{j+1}\right\rangle \quad \text { (by antisymmetry) } \\
& =-\left\langle\phi \circ \psi\left(w_{i+1}\right), w_{j+1}\right\rangle \quad \text { (by commutativity) } \\
& =-\left\langle\phi(0), w_{j+1}\right\rangle \quad\left(\text { since } T^{j} \text { annihilates } w_{i+1}\right) \\
& =0 .
\end{aligned}
$$

The proof of the theorem follows from this orthogonality relation and a generalization of the well-known result that an isotropic subspace of an inner product space does not exceed half the dimension of the space.

LemMA 3.2. Let $S_{1}, \ldots, S_{k}$ be mutually orthogonal subspaces of a nondegenerate inner product space $V$. Then

$$
\sum_{i} \operatorname{dim} S_{i} \leqslant(k / 2) \operatorname{dim} V
$$

Proof. Because the pairing is perfect, there is an isomorphism of the dual of $S_{j}$ with $V / S_{j}{ }^{\perp}$, so that

$$
\operatorname{dim} V=\operatorname{dim} S_{j}+\operatorname{dim} S_{j}^{\perp}
$$


Because $S_{i}$ and $S_{j}$ are orthogonal, there is an injection of $S_{i}$ in $S_{j}{ }^{\perp}$, so that $\operatorname{dim} S_{j}^{\perp} \geqslant \operatorname{dim} S_{i}$.

The last two relations imply that

$$
\operatorname{dim} V \geqslant \operatorname{dim} S_{j}+\operatorname{dim} S_{i},
$$

which inequality we sum over all pairs $(i, j)$ with $i<j$ to get

$$
\sum_{i<j}\left(\operatorname{dim} S_{i}+\operatorname{dim} S_{j}\right) \leqslant(k(k-1) / 2) \operatorname{dim} V .
$$

Since the sum can also be evaluated as

$$
\sum_{i<j}\left(\operatorname{dim} S_{i}+\operatorname{dim} S_{j}\right)=(k-1) \sum_{i} \operatorname{dim} S_{i}
$$

we have

$$
\sum_{i} \operatorname{dim} S_{i} \leqslant(k / 2) \operatorname{dim} V
$$

4. Bounds for higher weight. We now prove two analogues of Theorem 1.1 in the higher weight case. In what follows $n$ denotes the weight, $r$ denotes the highest Hodge level, $s$ the lowest, and $l=r-s$ the level itself (see $\$ 2$ ).

THEOREM 4.1. Let $H$ be a Hodge structure of level $l>1$, and let $V$ be an infinitesimal variation of $H$ which satisfies ND1. Then

$$
\operatorname{dim} V \leqslant h^{r-1, s+1} .
$$

THEOREM 4.2. Let $H$ be a Hodge structure level $l>1$ with $h^{r . s}>1$ and let $V$ be an infinitesimal variation of $H$ which satisfies both ND1 and ND2. Then

$$
\operatorname{dim} V \leqslant \frac{1}{2} h^{r . s} h^{r-1, s+1} .
$$

As we have noted in the Introduction, generic hypersurface variations are nondegenerate (Theorem 7.1), so that the restrictive hypothesis is not without geometric foundation. To see what is gained by the estimates, note that the fiber dimension of the horizontal bundle is rather much larger:

$$
\operatorname{dim}_{f} T_{h}=h^{r . s} h^{r-1 . s+1}+h^{r-1 . s+1} h^{r-2 . s+2}+\cdots+\frac{1}{2}\left(h^{t, t-1}\right)^{2}
$$

for $n$ odd, with $t=(n+1) / 2$, and

$$
\operatorname{dim}_{f} T_{h}=h^{r \cdot s} h^{r-1, s+1}+h^{r-1, s+1} h^{r-2, s+2}+\cdots+h^{t, t-1} h^{t-1, t-2}
$$

for $n$ even, with $t=n / 2+1$. As in the weight two case there are situations in which the bounds are attained geometrically. The image of moduli in periods for these families are once again components of the geometric locus.

EXAMPLE 4.3. Let $S$ be the natural versal family of hypersurfaces of dimension $n$ and degree $n+2$. Then the image of $S$ in $\Gamma \backslash D$ is a component of the geometric locus. To check that the example works, note that the canonical bundle of such hypersurfaces is trivial. Serre duality then implies that $\operatorname{dim} H^{1}(X, \Theta)=$ $\operatorname{dim} H^{n-1}\left(X, \Omega^{1}\right)$, so that $\operatorname{dim} S=h^{n-1.1}$. Local Torelli [10] and Theorem 4.1 then combine to yield the required conclusion. 
As we show in Remark 6.9, the nondegeneracy assumptions are necessary for bounds of the kind given above to hold: there exist degenerate variations whose dimension is approximately $\frac{1}{2} \operatorname{dim}_{f} T_{h}$. Like the variations of weight two of maximal dimension discussed in the Introduction, these are also homogeneous, this time for a product of groups of type $U(p, q)$. We do not know if these variations can arise geometrically. In any case, one can show using Schmid's Nilpotent Orbit Theorem [16] that their monodromy groups are quite special: If $N$ is a logarithm of monodromy, then $N^{2}=0$.

To give the proofs, we begin with a result which shows that a tangent vector $\phi$ in $T$ is determined entirely by its first potentially nonzero component, provided that ND1 holds:

LemMa 4.4. Let $V$ be a nondegenerate variation on a Hodge structure $H$ with highest level $r$ and consider the natural projection of $T$ to a subspace $T_{r}$ of $\operatorname{Hom}\left(G^{r}, G^{r-1}\right)$. Then $\operatorname{dim} T=\operatorname{dim} T_{r}$.

Proof. Write $\phi$ as $\phi_{r}+\phi_{r-1}+\cdots+\phi_{s-1}$, where $\phi_{k}$ is in $\operatorname{Hom}\left(G^{k}, G^{k-1}\right)$. It suffices to show that $\phi_{r}$ determines $\phi_{i}$ for $i<r$. Consider first the case in which $i=r-1$. Let $y$ be an element of $G^{r-1}$, and let $x$ in $G^{r}, \psi$ in $T$ be such that $\psi(x)=y$. By the nondegeneracy hypothesis this is possible. Then we have

$$
\phi_{r-1}(y)=\phi_{r-1}\left(\psi_{r}(x)\right)=\psi_{r-1}\left(\phi_{r}(x)\right),
$$

where we have used the commutativity (integrability) property of the infinitesimal variation. Since $\psi_{r-1}$ does not depend on $\phi, \phi_{r-1}$ is determined by $\phi_{r}$. Repeating the argument as many times as is necessary, we see that $\phi_{r}$ determines all of the "lower" components $\phi_{i}$, as required.

Proof of Theorem 4.1. By the lemma,

$$
\operatorname{dim} T=\operatorname{dim} T_{r} \leqslant \operatorname{dim} \operatorname{Hom}\left(G^{r}, G^{r-1}\right)=h^{r-1, s+1} .
$$

Proof of TheOREM 4.2. The argument is similar to the proof of Theorem 1.1 but requires slightly more effort to get the orthogonality relations. We begin as before. Let $w_{1}, \ldots, w_{m}$ be a basis for $G^{r}$, the Hodge component of highest level, and define a decreasing filtration of $T$ by

$$
T^{p}=\text { annihilator }\left(w_{1}, \ldots, w_{p}\right) .
$$

Define subspaces of $G^{r-1}$ by

$$
S_{k}=T^{k}\left(w_{k+1}\right)
$$

and note that there is an isomorphism

$$
T \cong\left(\bigoplus_{k} S_{k}\right) \oplus\left(T^{p}\right)
$$

where $T^{p}$ is the annihilator of $G^{r}$ in $T$. To eliminate $T^{p}$ we use the following result.

Lemma 4.5. Let $V(H)$ be an infinitesimal variation, let $G^{r}$ be the Hodge component of highest level, and let $A$ be the annihilator of $G^{r}$ in T. If $V$ satisfies $N D 1$ then $A=0$.

Proof. Let $\phi$ be an arbitrary element of $A$. We must show that $\phi$ annihilates $G^{r-j}$ for all $j \geqslant 0$. Because of the nondegeneracy assumption, $G^{r-j}$ is generated by the vectors $x=\psi^{1} \circ \psi^{2} \circ \cdots \circ \psi^{j}(y)$, where the $\psi^{\prime}$ 's are in $T$ and where $y$ is in $G^{r}$. But 
the commutativity property allows us to write

$$
\phi(x)=\phi\left(\psi^{1} \circ \psi^{2} \circ \cdots \circ \psi^{j}(y)\right)=\psi^{1} \circ \psi^{2} \circ \cdots \circ \psi^{j}(\phi(y))=0 .
$$

Since $\phi$ annihilates all components of $G^{\circ}$, it must be the zero element of $T$, as required.

We are now in a situation similar to that of Theorem 1.1:

$$
T \cong \bigoplus_{k} S_{k} \text {. }
$$

To conclude we must establish the appropriate analogue of the orthogonality conditions.

Lemma 4.6. Let $a=l-2$, where $l$ is the level. Then the subspaces $S_{i}$ and $T^{(a)}\left(S_{j}\right)$ are orthogonal.

Proof. Let $\phi_{i}$ be an element of $T^{i}, \phi_{j}$ an element of $T^{j}$, where $i<j$, and note that $\phi_{j}\left(w_{i+1}\right)=0$. Then one deduces the following relations:

$$
\begin{array}{rlrl}
\left\langle\phi_{i}\left(w_{i+1}\right), \psi_{1} \circ \psi_{2} \circ \cdots \circ \psi_{a} \circ \phi_{j}\left(w_{j+1}\right)\right\rangle & & \\
& =\left\langle\phi_{i}\left(w_{i+1}\right), \phi_{j} \circ \psi_{1} \circ \psi_{2} \circ \cdots \circ \psi_{a}\left(w_{j+1}\right)\right\rangle & & \text { (commutativity) } \\
& =-\left\langle\phi_{j} \circ \phi_{i}\left(w_{i+1}\right), \psi_{1} \circ \psi_{2} \circ \cdots \circ \psi_{a}\left(w_{j+1}\right)\right\rangle & & \text { (skew symmetry) } \\
& =-\left\langle\phi_{i} \circ \phi_{j}\left(w_{j+1}\right), \psi_{1} \circ \psi_{2} \circ \cdots \circ \psi_{a}\left(w_{j+1}\right)\right\rangle & & \text { (commutativity) } \\
& =0 & & \left(\phi_{j}\left(w_{i+1}\right)=0\right) .
\end{array}
$$

Lemma 4.7. Let $V, W$ be vector spaces over a field $k$ and let $\langle$,$\rangle be a perfect$ pairing of $V$ and $W$ to $k$. Let $S$ be a subspace of $V$, and let $S^{\perp}$ be its orthogonal complement in $W$. Then $\operatorname{dim} S+\operatorname{dim} S^{\perp}=\operatorname{dim} V$.

Proof. The induced pairing between $S$ and $W / S^{\perp}$ is perfect.

We can now complete the proof of Theorem 4.2. By Lemma 4.6 the subspaces $S_{i}$ and $T^{(c)}\left(S_{j}\right)$ are orthogonal, so that

$$
S_{i} \subset\left[T^{(a)}\left(S_{j}\right)\right]^{\perp} .
$$

From this and Lemma 4.7 one obtains the relations

$$
\operatorname{dim} G^{r}{ }^{1}=\operatorname{dim}\left[T^{(a)}\left(S_{j}\right)\right]^{\perp}+\operatorname{dim}\left[T^{(a)}\left(S_{j}\right)\right]^{\perp \perp} \geqslant \operatorname{dim} S_{i}+\operatorname{dim}\left[T^{(a)}\left(S_{j}\right)\right] .
$$

From the hypothesis ND2 we conclude that

$$
\operatorname{dim} S_{i}+\operatorname{dim} S_{j} \leqslant \operatorname{dim} G^{r-1} .
$$

Summing over all pairs $(i, j)$ with $i<j$ as in the proof of Theorem 1.1, we obtain

$$
\operatorname{dim} T=\sum \operatorname{dim} S_{i} \leqslant \frac{1}{2} \operatorname{dim} G^{r} \cdot \operatorname{dim} G^{r-1} .
$$

5. Examples (infinitesimal). We begin with an example of Harsh Pittie [15], an infinitesimal variation of Hodge structure which realizes the bound on the dimension given by Theorem 1.1 .

THEOREM 5.1. Fix a Hodge structure $H$ of weight two and let I be a totally isotropic subspace of $G^{1}$. Then there is an infinitesimal variation $V_{I}(H)$ with

$$
T=\operatorname{Hom}\left(G^{2}, I\right) \quad \text { and } \delta: T \rightarrow E^{-1}(H)
$$

injective. 
REMARKS 5.2. (1) If $h^{2,0}>1$ and if $\operatorname{dim} G^{1}$ is even, then $\operatorname{dim} V_{i}(H)=\frac{1}{2} h^{2.0} h^{1.1}$, so that the bound of Theorem 1.1 is attained.

(2) An infinitesimal variation such as the one just given is totally isotropic: by definition, the image of $G^{2}$ in $G^{1}$ under $T$ is totally isotropic.

(3) A corollary of the proof below is that $V$ is totally isotropic if and only if $\phi \circ \psi=0$ for all $\phi, \psi$ in $T$. This strong orthogonality property is quite special (indeed, rare).

Proof. Let $I$ be a totally isotropic subspace of $G^{1}$, and observe that for each $\phi$ in $\operatorname{Hom}\left(G^{2}, I\right)$, that the transpose ${ }^{t} \phi$, which is a map from $G^{1}$ to $G^{0}$, kills $I$. Indeed, if $x$ is in $G^{2}$ and $a$ is in $I$, then

$$
\left\langle{ }^{t} \phi(a), x\right\rangle=-\langle a, \phi(x)\rangle=0,
$$

since both $a$ and $\phi(x)$ are in $I$. Let $T$ be $\operatorname{Hom}(G, I)$ and define a homomorphism

$$
\delta: T \rightarrow E^{-1}(H)
$$

by setting $\psi=\delta(\phi)=\phi-{ }^{t} \phi$. Because any two image vectors $\psi, \psi^{\prime}$ satisfy $\psi \circ \psi^{\prime}=0$, they must satisfy, a fortiori, the commutation relations $\left[\psi, \psi^{\prime}\right]=0$.

The next result, which is due to Mark Green [8], shows that Pittie's variation is essentially unique.

THEOREM 5.3 (INFINITESIMAL RIGIDITY). Let $V$ be an infinitesimal variation of $a$ Hodge structure $H$ of weight two with $h^{2,0}>2$. If $\operatorname{dim} V=\frac{1}{2} h^{2,0} h^{1,1}$, then $h^{1.1}$ is even and $V$ is of the form $V_{I}(H)$ for some choice of maximal totally isotropic subspace $I$.

Corollary 5.4. An infinitesimal variation of weight two of maximal dimension with $h^{2 .()}>2$ is totally isotropic.

Proof OF THE THEOREM. Suppose that $\operatorname{dim} T=\frac{1}{2} h^{2,0} h^{1,1}$. Then we have

$$
\sum \operatorname{dim} S_{i}=\frac{1}{2} h^{2,0} h^{1,1} \geqslant \frac{3}{2} h^{1,1},
$$

where we have used the hypothesis on $h^{2.0}$ to obtain the inequality. If for all pairs $i<j$ we have

$$
\operatorname{dim} S_{i}+\operatorname{dim} S_{j}<h^{1,1},
$$

then the argument used in the proof of Theorem 1.1 gives

$$
\sum \operatorname{dim} S_{i}<\frac{1}{2} h^{2.0} h^{1,1}
$$

contradicting $(*)$. Consequently there exists a pair $i<j$ such that

$$
\operatorname{dim} S_{i}+\operatorname{dim} S_{j} \geqslant h^{1.1} .
$$

Since the pairing on $G^{1}$ is perfect, $\operatorname{dim} S_{j}+\operatorname{dim} S_{j}{ }^{\perp}=h^{1,1}$. Subtracting this relation from the preceding inequality, one obtains $\operatorname{dim} S_{i} \geqslant \operatorname{dim} S_{j}^{\perp}$. But the orthogonality relations give $S_{j}{ }^{\perp} \supset S_{i}$, so that $\operatorname{dim} S_{j}{ }^{\perp} \geqslant \operatorname{dim} S_{i}$. From these relations one sees that $S_{i}=S_{j}^{\perp}$.

If $\operatorname{dim} S^{k}<\frac{1}{2} h^{1,1}$ for the remaining $k$ different from $i, j$, then $(*)$ is once again violated. Consequently there is an index $k \neq i, j$ such that $\operatorname{dim} S_{k} \geqslant \frac{1}{2} h^{1.1}$. The orthogonality conditions give $S_{k} \subset S_{i}{ }^{\perp}$, from which one obtains $\operatorname{dim} S_{i}{ }^{\perp} \geqslant \frac{1}{2} h^{1.1}$ and 
$\operatorname{dim} S_{i} \leqslant \frac{1}{2} h^{1.1}$. This last inequality combined with analogous relation for $S_{j}$ and with (*) implies that $\operatorname{dim} S_{i}=\operatorname{dim} S_{j}=\frac{1}{2} h^{1,1}$. These equalities combined with the relations $S_{i}=S_{j}{ }^{\perp} \supset S_{k}$ and $\operatorname{dim} S_{k} \geqslant \frac{1}{2} h^{1.1}$ imply that $S_{i}=S_{j}{ }^{\perp}=S_{k}$.

From this last fact one deduces that $S_{i}=S_{k} \subset S_{i}{ }^{\perp}$, so that $S_{i}$ is an isotropic (and therefore maximal isotropic) subspace of $G^{1}$, which we henceforth call $I$. But then the remaining spaces $S_{t}$, which are orthogonal to $I=S_{i}$, must be contained in $I^{\perp}=I$. Consequently $T$ is contained in $\operatorname{Hom}\left(G^{2}, I\right)$. The hypothesis on the dimension now implies that $T$ is equal to $\operatorname{Hom}\left(G^{2}, I\right)$, as required.

REMARKS (5.5). (a) Consider two infinitesimal variations of the above form, $V_{I_{1}}(H)$ and $V_{I_{2}}(H)$. Let $f$ be an automorphism of $G^{1}$ which carries $I_{1}$ to $I_{2}$ and which preserves the bilinear form on $G^{1}$. Such an automorphism exists and induces an automorphism of $\operatorname{Hom}\left(G^{2}, G^{1}\right)$ which carries $\operatorname{Hom}\left(G^{2}, I_{1}\right)$ to $\operatorname{Hom}\left(G^{2}, I_{2}\right)$. Thus, any two maximal totally isotropic variations are conjugate, and as such have no moduli. This, with Corollary 5.4, shows that infinitesimal variations of maximal dimension are unique up to conjugation, provided that $h^{2,0}>2$.

(b) When $h^{2,0}=2$ the dimension of a maximal infinitesimal variation is $h^{1,1}$. To see that this is so, let $T$ be a $k$-dimensional commutative subspace of $E^{-1}$ and let $\mathscr{Z}(T)$ be its centralizer. If $A_{1}, \ldots, A_{k}$ give a basis for $T$, then $\mathscr{Z}(T)$ is defined by the $k$ equations $\left[A_{i}, X\right]=0$. But $\left[E^{-1}, E^{-1}\right] \subset E^{-2}$, and in our case $E^{-2}$ has dimension one, so each of the preceding equations imposes one linear condition. Consequently $\operatorname{dim} \mathscr{Z}(T) \geqslant 2 h^{1.1}-k$. However, if $T$ is maximal, then $T=\mathscr{Z}(T)$, so that $k \geqslant 2 h^{1.1}-k$, and hence $k \geqslant h^{1.1}$. But the upper bound of Theorem 1.1 gives $k \leqslant h^{1.1}$, from which the claim follows.

(c) One may show by explicit computation that neither of the infinitesimal variations of Examples $1.2-1.3$ is totally isotropic. Consequently neither can be of the form $V_{l}(H)$. Indeed, the infinitesimal variations of these examples have moduli à la Donagi's proof of generic Torelli [6], whereas the $V_{I}(H)$ have none.

(d) The amount of isotropy present in an infinitesimal variation is a controlling factor in bounding the a priori dimension. To see why this is so, observe that if the subspaces $S_{i}$ used in the proof of Theorem 1.1 are nonisotropic, then they are complementary as well as orthogonal. In this case one obtains the stronger estimate $\operatorname{dim} V \leqslant h^{1.1}$.

(e) A trivial case in which anisotropy is forced is the case $h^{1.1}=1$. Such variations satisfy the bound $\operatorname{dim} V \leqslant 1$. To see that this is so, introduce bases for the $G^{p}$ in order to write tangent vectors $a, b$ as row vectors of length $g$. The integrability conditions are then expressed by the matrix equation

$$
[a, b]={ }^{t} a b-{ }^{t} b a=0 .
$$

This forces $a_{i} b_{j}-a_{j} b_{i}=0$, so that $a$ and $b$ are dependent, as required.

(f) Little seems known about the isotropy properties of geometric variations. For the Fermat surfaces the variation is neither anisotropic nor totally isotropic relative to a monomial basis for $G^{2}$.

6. Examples (global). The next result shows that there are actual-not merely infinitesimal-variations of Hodge structure which satisfy the bound of Theorem 
1.1. The associated infinitesimal variations are (as they must be for $h^{2.0}>2$ ) totally isotropic.

THEOREM 6.1. Let $H$ be a Hodge structure of weight two with $h^{1,1}$ even. Let $m=\frac{1}{2} h^{2,0} h^{1.1}$. Then there exists an open neighborhood $U$ of the origin in $\mathbf{C}^{m}$ and an injective horizontal holomorphic map $v: U \rightarrow D$ which takes 0 to $H$. Moreover, if $H_{q}$ denotes the Hodge structure corresponding to $q$ in $U$ under $v$, then

$$
\left(H_{q}, f_{q^{*}}\right)=V_{I}\left(H_{q}\right)
$$

for some maximal isotropic subspace $I \subset G^{1}$.

The proof of the theorem rests on a distinguished choice of isotropic subspace of $H_{\mathrm{C}}$, a choice which we shall have occasion to use further.

Lemma 6.2. Let $H$ be a weight two Hodge structure with $h^{1,1}$ even. Then there is a maximal isotropic subspace $K$ of $H_{\mathbf{C}}$ with

$$
H_{\mathbf{C}}=K \oplus \bar{K}
$$

and with $I=K \cap H^{1,1}$ maximal isotropic in $H^{1,1}$.

Proof. Let $I$ be a maximal isotropic subspace of $H^{1,1}$. Now $A=I \cap \bar{I}$ is the complexification of a real subspace $A_{\mathbf{R}}$ and $\langle$,$\rangle is negative definite on \left(H^{1,1}\right)_{\mathbf{R}}$. However, by the isotropy assumption, $\langle$,$\rangle vanishes on A_{\mathbf{R}}$, so that $A_{\mathbf{R}}$, and hence $A$ as well, must vanish. We conclude that $I \cap \bar{I}=0$, so that there exists a direct decomposition $H^{1,1}=I \oplus \bar{I}$. Next define a maximal isotropic subspace of $H_{\mathbf{C}}$ by $K=H^{2,0} \oplus I$. This choice of $K$ fulfills the requirements of the lemma.

Proof OF THEOREM 6.1. Given a subspace $S$ of $K$ define a decreasing filtration of $H_{\mathbf{C}}$ by

$$
\left[F^{0} \supset F^{1} \supset F^{2}\right]=\left[H_{\mathbf{C}} \supset S^{\perp} \supset S\right] .
$$

Because of the position of $S$, this makes sense:

$$
S \subset K=K^{\perp} \subset S^{\perp} .
$$

Now consider the Grassmannian

$$
\text { Grass }=\{S \subset K \mid \operatorname{dim} S=a\} .
$$

When $S=S_{0}$ is the $(2,0)$ subspace of the reference structure used to define $K$, the filtration defined above is the reference Hodge filtration and so

$$
F^{2} \cap \bar{F}^{2}=0, \quad F^{1} \cap \bar{F}^{2}=0 .
$$

Since these relations remain true for small perturbations, $F^{*}(S)$ defines a Hodge structure for $S$ in an open neighborhood $U$ of $S_{0}$. Since the positivity conditions which define a polarized Hodge structure also remain true under small perturbations, we conclude that the map $S \mapsto F^{*}(S)$ defines a variation of Hodge structure $v: U \rightarrow D$.

To see that the associated infinitesimal variation is as claimed, consider a curve in $U$ defined by

$$
S(t)=\operatorname{span}\left(v_{1}(t), \ldots, v_{g}(t)\right),
$$


where $S(0)=S_{0}$ defines the reference filtration. The derivative of the associated curve of Hodge filtrations, evaluated at $t=0$, is the homomorphism

$$
\varphi: S_{0} \rightarrow S_{0}^{\perp} / S_{0}
$$

determined by

$$
v_{j}(0) \mapsto v_{j}^{\prime}(0)
$$

Since the vector-valued function $v(t)$ may take arbitrary values in the subspace $K$ of $H_{\mathrm{C}}$, the homomorphism $\varphi$ may take arbitrary values in the totally isotropic quotient space, $K / S_{0} \cong I$.

By Theorem 5.3 infinitesimal variations of maximal dimension with $h^{2.0}>2$ are rigid; they must be of the form $\operatorname{Hom}\left(F^{r}, I\right)$, hence are conjugate under the action of the real Lie group which acts on the classifying space. Variations of maximal dimension also satisfy a rigidity property.

ThEOREM 6.3 (LOCAL RIGIDITy). Let $m=\frac{1}{2} h^{2.0} h^{1.1}$, let $U$ be a simply-connected neighborhood of 0 in $\mathbf{C}^{m}$, and let $v: U \rightarrow D$ and $v^{\prime}: U \rightarrow D$ be variations of Hodge structure of weight two of dimension $m$. If $h^{2.0}>2$, then there is an element $g$ of $G=\operatorname{Aut}(D)$ such that the relation $g v=v^{\prime}$ holds on some neighborhood $U_{0}$ of 0 in $U$.

Proof. Let $v$ be a local variation of maximal dimension. It suffices to show that the subspace $K(z)$ is constant. To this end we observe that the filtration below defines what for all practical purposes is a variation of Hodge structure of weight three:

$$
\left[L^{3} \subset L^{2} \subset L^{1} \subset L^{0}\right]=\left[F^{2} \subset K \subset F^{1} \subset F^{0}\right]
$$

Certainly the $L$ filtration defines an unpolarized Hodge structure, since

$$
L^{3} \oplus \bar{L}^{1}=F^{2} \oplus \bar{F}^{1}, \quad L^{2} \oplus \bar{L}^{2}=K \oplus \bar{K} .
$$

To show that the family of $L$ filtrations defines a generalized infinitesimal variation of Hodge structure, observe that if $\varphi$ is in $T$, then $\varphi\left(L^{3}\right)$ lies in $L^{2}$ by hypothesis. To see that $\varphi\left(L^{2}\right)$ lies in $L^{1}$, note that $\langle\varphi(x), y\rangle=-\langle x, \varphi(y)\rangle$. If $x \in L^{3}$ and $y \in L^{2}$, then the left-hand member of the equality is an inner product of elements of $K$, hence is zero. Since the right-hand member must vanish for all $x \in L^{3}=F^{2}$, $\varphi(y)$ must lie in the orthogonal complement of $F^{2}$, i.e. in $F^{1}=L^{1}$, as required. Thus, the $L$ filtration gives what looks like a polarized variation of Hodge structure, except that the bilinear form is symmetric rather than antisymmetric, as it should be in weight three.

We now use the integrability conditions for this variation to show that $K=L^{2}$ must be constant. To this end, let $\left\{e_{i}\right\},\left\{f_{j}\right\},\left\{g_{k}\right\}$ be bases for $G^{3}, G^{2}, G^{1}$, respectively, where now $G^{p}$ refers to a graded quotient of the $L$ filtration. The "same" bases with superscripts will be used to denote the dual bases. By hypothesis there exists a basis $\left\{\varphi_{i j}\right\}$ of $T$ such that the component which maps $G^{3}$ to $G^{2}$ is $e^{i} \otimes f_{j}$. These are the "standard" rank one elements of $\operatorname{Hom}\left(G^{3}, G^{2}\right)$ represented by matrices which have a single nonzero element. Let us write the components of $\varphi_{i j}$ as $\alpha_{i,}, \beta_{i,}, \gamma_{i j}$, where the decomposition is the natural one determined by $G^{\bullet}: \alpha_{i j}$ maps 
$G^{3}$ to $G^{2}$, etc. Write $\beta_{i j}$ as below:

$$
\beta_{i j}=\sum_{r s} M_{i j r s} f^{r} \otimes g_{s} .
$$

The $\beta$ component of the commutator $\left[\varphi_{i j}, \varphi_{k l}\right]$ is

$$
\beta_{i j} \circ \alpha_{k l}-\beta_{k l} \circ \alpha_{i j}=\sum_{s}\left(M_{i j l s} e^{k} \otimes g_{s}-M_{k l j s} e^{i} \otimes g_{s}\right)=0 .
$$

Because $\operatorname{dim} G^{3}=h^{2,0}>1$ there exists, for each pair $(i, j)$ and for each $l$ a pair $(k, l)$ with $i \neq k$. Since the set

$$
\left\{e^{i} \otimes g_{1}, e^{i} \otimes g_{2}, e^{i} \otimes g_{3}, \ldots, e^{k} \otimes g_{1}, e^{k} \otimes g_{2}, e^{k} \otimes g_{3}, \ldots\right\}
$$

is linearly independent, the coefficients $M_{i j l s}$ must vanish for all $(i, j, l, s)$. Therefore $\beta_{i j}$ itself vanishes. But then the $L^{2}$-to- $L^{1}$ component of the basis vectors $\phi_{i j}$ vanishes, so that $K=L^{2}$ has derivative zero, as required.

We now show that the variation Hodge structure just defined is the restriction of one which is homogeneous for an action of $U(p, q)$ where

$$
p=h^{2,0}, \quad q=h^{1,1} / 2, \quad m=p q=\frac{1}{2} h^{2.0} h^{1,1} \quad \text { (maximal dimension) }
$$

and where $h^{1,1}$ is even by hypothesis. To begin, recall that the generalized ball

$$
B=\left\{Z \mid Z=p \times q \text { complex matric with }{ }^{t} \bar{Z} Z<I_{q}\right\}
$$

is a space of dimension $m$ which is homogeneous for $U(p, q)$. To describe the action, write a matrix $M$ in $U(p, q)$ in block form $\left[\begin{array}{ll}A & B \\ C & D\end{array}\right]$, where $A$ has size $p \times p$ and $D$ has size $q \times q$, set

$$
M Z=(A Z+B)(C Z+D)^{-1},
$$

and note that the isotropy group of the zero matrix can be identified with $U(p) \times U(q)$.

THEOREM 6.4 (EXISTENCE OF UNITARY VARIATIONS). There is a homomorphism $\phi: U(p, q) \rightarrow S O(2 p, 2 q, \mathbf{R})$ which induces an equivariant, holomorphic, and horizontal map of homogeneous spaces $v: B \rightarrow D$. The resulting variation of Hodge structure is totally isotropic and of maximum possible dimension, $p q=\frac{1}{2} h^{2,0} h^{1,1}$.

COROllaRY 6.5 (QUASIPROJECTIVE VARIATIONS OF MAXIMAL DIMENSION). If $h^{1,1}$ is even, then there exists a variation of Hodge structure $v: X \rightarrow \Gamma \backslash D$ of dimension $\frac{1}{2} h^{2,0} h^{1,1}$ with $X$ quasiprojective (even projective).

Proof of 6.5. We note $[1,2]$ that there are discrete subgroups $\Gamma$ of $U(p, q)$ which act on $B$ with quasiprojective (even projective [3]) quotient. It suffices to choose $X=\Gamma \backslash B$, then to define the action of $\Gamma$ on $D$ via the homomorphism $\varphi$ : $U(p, q) \rightarrow G$.

Proof of 6.4. Let $H_{\mathbf{R}}$ be a real vector space of dimension $2 n=2(p+q)$ endowed with a symmetric bilinear form $Q$ of signature $(2 p, 2 q)$ and let $D$ be the space of all $Q$-polarized real Hodge structures with $h^{2,0}=p$ and $h^{1,1}=2 q$. Let $G$ be the connected component of the identity of the group of linear automorphisms of $H_{\mathbf{R}}$ which preserve $Q$. Fix a reference Hodge structure $H_{0}$, let $V$ be the isotropy group of the reference structure, and recall [9] that the orbit map $g \mapsto g H_{0}$ defines 
an isomorphism $G / V \rightarrow D$. Consider the decomposition

$$
H_{\mathbf{C}}=K \oplus \bar{K}
$$

given by Lemma 6.2, let $M$ be its isotropy group, and let $W$ be the intersection of $m$ with $V$. We claim that the map of homogeneous spaces given by $M / W \rightarrow G / V$ fulfills the requirements of the theorem. Indeed, the map $g \mapsto g \mid K$ defines an isomorphism of $M$ with $U(p, q)$ viewed as a group of automorphisms of $K$, and the restriction of this map defines an isomorphism of $W$ with $U(p) \times U(q)$, so that $M / W$ is naturally identified with $B$. Since a Hodge structure in the image of $v$ admits arbitrarily small perturbations subject to the condition that $F^{2} \subset K$, it is clear that $v$ is locally of the form given by Theorem 6.1 , hence infinitesimally of the form given by Theorem 5.1.

REMARK 6.6 (GlobAL RIGIDITY). Let $v: S \rightarrow \Gamma \backslash D$ be a weight two variation of maximal dimension $m=\frac{1}{2} h^{2,0} h^{1,1}$ with $h^{2,0}>2$. The local rigidity Theorem 6.3 implies that $v$ must be a discrete quotient of a $U(p, q)$-homogeneous variation associated to a maximal isotropic subspace $K$.

REMARK 6.7 (ARITHMETIC QUOTIENTS). One can in fact give an explicit construction of quasiprojective variations which satisfy the bound of Theorem 1.1. To do so, let $H_{\mathrm{Z}}$ be a lattice with an orthogonal involution $J$. Such objects certainly exist. Let $H_{\mathbf{Z}}=\mathbf{Z}^{2 n}$, where $2 n=2(p+q)$, let $I_{r}$ be the identity matrix of rank $r$, let $J_{r}$ be the standard symplectic matrix of rank $2 r$, let the polarizing form $Q$ be the direct sum of $I_{2 p}$ and $-I_{2 q}$, and let $J$ be the direct sum of $J_{2 p}$ and $J_{2 q}$. Given such an involution there is a natural splitting

$$
H_{\mathbf{C}}=K \oplus \bar{K}
$$

where $K$ and $\bar{K}$ are the $i$ and $-i$ eigenspaces of $J$, respectively. Let $G(X)$ be the subgroup of $\operatorname{Sl}\left(H_{\mathbf{R}}\right)$ which preserves the structure $X$. If $H$ is a reference Hodge structure with $H^{2,0}$, contained in $K$, then we have

$$
\begin{aligned}
& G=G(Q) \cong S O(2 p, 2 q), \quad H=G(Q, J) \cong U(p, q), \\
& V=G(Q, H) \cong U(p) \times S O(2 q), \quad W=G(Q, J, H) \cong U(p) \times U(q), \\
& D=G / V, \quad M=H / W .
\end{aligned}
$$

Moreover, the subgroup $\Gamma=G\left(Q, J, H_{\mathbf{z}}\right)$ of $G(Q, J)$ is arithmetic, so that the quotient $\Gamma \backslash M / W$ is quasiprojective $[1,2]$, as required.

REMARK 6.8 (ARITHMETIC CONSEQUENCES OF TOTAL ISOTROPY). As we have just seen, there are totally isotropic variations whose base space is an algebraic variety and whose monodromy group is arithmetic. However, the isotropy condition places severe restrictions on the monodromy. The first of these follows immediately from hypothesis: $\Gamma$ preserves a proper subspace $(K)$ of $H_{\mathbf{C}}$ and so is reducible over the complex numbers (although not over the reals). The second is a restriction on the possible logarithms of monodromy transformations in $\Gamma$. Let $v_{0}: \Delta^{*} \rightarrow \Gamma \backslash D$ be a one-variable degeneration deduced from $v$, let $g$ be the associated monodromy transformation, let $n$ be the least integer such that $g^{n}$ is unipotent, and let $N=\log g^{n}$. Then $N^{2}=0$ (as if the variation were of weight one). To see that this is 
so, apply Schmid's nilpotent orbit theorem [16] to $v_{0}$ to write

$$
F^{2}(t)=\exp [(1 / 2 \pi i)(\log t) N] \Phi(t)
$$

for some holomorphic function $\Phi$ from $\Delta$ to $D$. The second derivative of $F^{2}(t)$ is congruent to the expression below, modulo $F^{1}(t)$ and modulo terms which have slower growth in $1 /|t|$ :

$$
\left(-1 / 2 \pi i t^{2}\right) N^{2} \exp [(1 / 2 \pi i)(\log t) N] \Phi(t) .
$$

Thus, if $N^{2} \neq 0$, the second derivative of $F^{2}$ will be nonzero for $t$ sufficiently small, contrary to the assumption of total isotropy, which requires $\phi^{2}=0$.

Remark 6.9 (More unitary Variations of Hodge STRUCTURe). A weight three variation: Consider a weight three Hodge structure $H_{0}$, and let $B$ be the subset of the corresponding classifying space such that $F^{2} \subset F_{0}^{2}$. Since the Hermitian form associated to the polarizing form has signature $(p, q)=\left(h^{3,0}, h^{2,1}\right)$ on $F_{0}^{2}$, the isotropy group $G\left(Q, F_{0}^{2}\right)$ can be identified with $U(p, q)$. Since one can also identify the isotropy group $G\left(Q, F_{0}^{2}, H\right)$ with $U(p) \times U(q)$, the subset $B$ is identified with the generalized ball viewed as a $U(p, q)$-homogeneous space. The resulting variation of Hodge has dimension $p q$, so it satisfies the bound of Theorem 5.1 but violates that of 5.2 if $p>1$. However, we observe that the variation is degenerate, since $T$ is of the form

$$
T=\left\{\varphi \text { in } E^{-1}(H) \mid \varphi_{2}=0\right\},
$$

where $\varphi_{2}$ is the component of $\varphi$ which maps $G^{2}$ to $G^{1}$. Nonetheless, the variation is maximal since it is infinitesimally maximal. Recall that the centralizer of an infinitesimal variation $(\$ 2)$ is defined by

$$
\mathscr{Z}(T)=\left\{\psi \text { in } E^{-1}(H) \mid[\psi, \varphi]=0 \text { for all } \varphi \text { in } T\right\},
$$

and that $V$ is maximal if and only if $T=\mathscr{Z}(T)$. In our case the commutation relations $[\psi, \varphi]=0$ give

$$
\psi_{2} \circ \varphi_{3}=\varphi_{2} \circ \psi_{3}=0
$$

where $\varphi \in T$ and $\psi \in \mathscr{Z}(T)$. Since $\varphi_{3}$ is surjective, $\psi_{2}$ must vanish, so that $\psi$ is in $T$. Thus $\mathscr{Z}(T)=T$, as required.

$A$ weight four variation: The same technique allows one to construct a maximal weight four variation with $F^{3}$ and $F^{2}$ fixed. Once again the variation is $U(p, q)$-homogeneous with $(p, q)=\left(h^{4,0}, h^{3,1}\right)$. The infinitesimal variation is characterized by the equations $\varphi_{3}, \varphi_{2}=0$ and so is degenerate. A calculation of the centralizer similar to that given above shows that the variation is maximal.

$A$ weight five variation: Let $(p, q, r)=\left(h^{5,0}, h^{4,1}, h^{3.2}\right)$. Fix a reference Hodge structure $H_{0}$ and consider the set $X$ of all structures with $F^{4}$ and $F^{2}$ fixed. Let $g$ be an element in the stabilizer of $X$, and observe that the restriction of $g$ to the direct sum of $F^{4}$ and $F^{2} / F^{4}$ induces an isomorphism of the stabilizer with $U(p, q) \times$ $\mathrm{Sp}(r)$. Under the map the isotropy group of the reference structure corresponds to $U(p) \times U(q) \times U(r)$. There results an identification of $X$ with $B \times \mathscr{H}$, where $B$ is the generalized ball and $\mathscr{H}$ is the Siegel upper half space of genus $r$. Tangent 
vectors to $X$ are horizontal, so that $X$ is indeed a variation of Hodge structure-one of dimension $p q+\frac{1}{2} r(r-1)$. It is, as one expects, degenerate, since the infinitesimal variation is characterized by $\varphi_{4}=0$. Again, one shows that $T$ is its own centralizer, so that $V$ is maximal.

Weight greater than five: By taking direct sums of appropriately spaced (and therefore commuting) variations, as we have done in the preceding example, one may construct maximal but degenerate variations of dimension roughly $\frac{1}{2} \operatorname{dim}_{f} T_{h}$. These variations are homogeneous for a product of groups of type $U(p, q)$, with possibly one factor of type $\operatorname{Sp}(r)$. We conjecture that the maximal dimension of an infinitesimal variation with fixed Hodge numbers is that of a homogeneous variation of the kind discussed above, so that the maximal dimension is always roughly $\frac{1}{2} \operatorname{dim}_{f} T_{h}$. We also conjecture that variations of maximal dimension, modulo special cases, are both degenerate and unique.

7. Nondegeneracy. We now show that the nondegeneracy hypotheses made for Theorems 5.1 and 5.2 are nonvacuous.

THEOREM 7.1. Let $V(X)$ be the infinitesimal variation defined by a smooth hypersurface in projective space. Then $V(X)$ satisfies $N D 1$. If in addition $X$ is generic, then $V(X)$ satisfies $N D 2$ as well.

The proof rests on the identification of the primitive cohomology of $X$ with polynomials via Griffiths' generalization of the Poincaré residue [10], the essentials of which we now recall. Let $Z_{0}, \ldots, Z_{n+1}$ be homogeneous coordinates for the ambient projective space, let $d V=d Z_{0} \wedge \cdots \wedge d Z_{n+1}$, let

$$
E=\sum \partial / \partial Z_{k}
$$

be the Euler vector field, and let $\Omega=\langle E, d V\rangle$ be the contraction of $E$ with $\Omega$. Consider the expressions $\Omega_{A}=A \Omega / Q^{r}$, where $Q$ is a defining polynomial for $X$. If $\Omega_{A}$ is homogeneous of degree zero with respect to the natural $\mathbf{C}^{*}$ action, then it defines a rational $(n+1)$-form on $\mathbf{P}^{n+1}$ with a pole of order $r$ on $X$. This happens when the degree of $A$ is $a(r)=r d-(n+2)$, where $d$ is the degree of $Q$. The residue of $\Omega_{A}$ on $X$ is a cohomology class of dimension $n$ and Hodge level $n-r+1$ : the pole filtration is compatible, up to a suitable reindexing, with the Hodge filtration. Thus, if $S$ is the polynomial ring in the variables $Z_{0}, \ldots, Z_{n+1}$, then there is a composed map

$$
S^{u(r)} \rightarrow\left\{\Omega_{A} \mid \text { pole order } r\right\} \rightarrow F^{n-r+1} H^{n}(X)
$$

given by

$$
A \mapsto \Omega_{A} \mapsto \operatorname{residue}\left(\Omega_{A}\right) .
$$

Now rational forms of pole order $r$ which differ from forms of pole order $r-1$ by an exact form must map via the residue to $F^{n-r+2}$ rather than to $F^{n-r+1}$. One shows that this happens if and only if the coefficient polynomial $A$ lies in the Jacobian ideal of the defining equation:

$$
J(Q)=\operatorname{ideal}\left(\partial Q / \partial Z_{0}, \ldots, \partial Q / \partial Z_{n+1}\right) .
$$


Thus, if one defines the Jacobian ring of $Q$ to be the quotient ring $R=S / J(Q)$, then there is an induced map

$$
R^{a(q)} \rightarrow G^{n-q+1} H^{n}(X)_{0},
$$

where $G^{p}=F^{p} / F^{p-1}$. One of the fundamental results of [10] asserts that $(*)$ is an isomorphism.

Now let $H^{1}(X, \Theta)$ be the space of infinitesimal deformations of $X$. According to [10] there is an identification of this group with the $d$ th homogeneous component of the Jacobian ring via

$$
P \mapsto \text { deformation class at zero of the pencil }(Q+t P) .
$$

This identification is compatible with the preceding ones in the sense that the diagram below is commutative.

$$
\begin{array}{cccc}
R^{d} \times R^{a(k)} & \rightarrow & R^{d+a(k)} \\
\downarrow & & & \downarrow \\
H^{1}(\Theta) \times G^{n-k} & \rightarrow & G^{n-k-1}
\end{array}
$$

Furthermore there is a compatibility with the topological cup-product [4]: (i) there is a largest integer top such that $\operatorname{dim} R^{\text {top }} \neq 0$, (ii) $R^{\text {top }}$ has dimension one, (iii) if $p+q=$ top then the pairing

$$
R^{p} \times R^{q} \rightarrow R^{\mathrm{top}}
$$

given by multiplication is perfect, and (iv) this multiplication can be identified with the cup-product of residues of rational differential forms for appropriate $p$ and $q$. The integer top is given by $(d-2)(n+2)$. With these preliminaries complete, we proceed to the proof.

PROOF OF ND1. The assertion to be proved is equivalent to surjectivity of the top arrow in $(* *)$, which fact is clear.

PROOF OF ND2. This requires more work. Our argument is to first reduce the assertion to one about the Hilbert function of certain ideals, then to use a result of Macaulay [13] to further reduce to a statement about monomial ideals. Elementary arguments on the geometry of lattices then complete the proof.

To begin, recall that the assertion to be proved is that if $H$ is a Hodge structure of highest level $r$ and weight $n$ coming from a smooth hypersurface, and if $U$ is a subspace of $G^{r-1}$, then

$$
\operatorname{dim} T^{(r-s-2)} U \geqslant \operatorname{dim} U,
$$

where $r+s=n$. Because of the identification of $G^{\circ}$ with a part of $R^{\circ}$ which was discussed above, this last assertion is equivalent to

$$
\operatorname{dim} R^{d(r-s-2)} U \geqslant \operatorname{dim} U,
$$

where now $U$ is an arbitrary subspace of $R^{a(r-1)}$. It therefore suffices to prove the following.

Lemma 7.2. Let $R$ be the Jacobian ring of a generic hypersurface of degree $d$ and dimension $n$. Let $p$ be an integer in the range $(d$, top $/ 2)$ and let $q$ be such that $p+q=$ top. Let $I$ be an ideal of $R$ generated in degree $p$. Then $\operatorname{dim} I^{q} \geqslant \operatorname{dim} I^{p}$. 
The first step in the proof is to prove a special case.

Lemma 7.3. Let $R$ be the Jacobian ring of the Fermat hypersurface of degree $d$ and dimension $n$. Let $p$ be an integer in the range $(d$, top $/ 2)$ and let $q$ be such that $p+q=$ top. Let $I$ be an ideal of $R$ generated in degree $p$. Then $\operatorname{dim} I^{q} \geqslant \operatorname{dim} I^{p}$.

Proof. According to a result of Macaulay [13] the Hilbert function of a homogeneous ideal $I$ is the same as the Hilbert function of a certain ideal generated by monomials, the ideal of initial forms MI:

$$
\operatorname{dim} I^{m}=\operatorname{dim}(M I)^{m} .
$$

To define this ideal, choose a total ordering $(>$ ) of monomials which

(i) refines the partial order by degree,

(ii) is multiplicative: for monomials $a, b, m, a>b$ implies $m a>m b$.

The "lexicographic" ordering satisfies these conditions. Thus, for the $\operatorname{ring} k[x, y]$, one has

$$
x>y, \quad x^{2}>x y>y^{2}, \quad x^{3}>x^{2} y>x y^{2}>y^{3}, \quad \text { etc. }
$$

The initial form $M(P)$ of a homogeneous polynomial $P$ is the monomial of highest order which appears in $P$ with nonzero coefficient, and

$$
M I=\text { ideal generated by }\{M(P) \mid P \text { a form in } I\} \text {. }
$$

The initial forms of a generating set for $I$ appear in a generating set for $M I$ but additional monomials may appear as well. A special case is the Jacobian ideal of the Fermat hypersurface, for which we have $J=M J$, since $J$ is already generated by monomials. Let $I_{0}$ be the inverse image of $I$ in $S$ and apply Macaulay's result to get

$$
\operatorname{dim}(I)^{m}=\operatorname{dim}\left(I_{0}\right)^{m}-\operatorname{dim} J^{m}=\operatorname{dim}\left(M I_{0}\right)^{m}-\operatorname{dim}(M J)^{m}=\operatorname{dim}(M I)^{m},
$$

where $M I$ is the ideal of $R$ generated by initial forms of $I_{0}$. It therefore remains to show, as we do next, that the result is true for ideals generated by monomials, where $R$ is the Jacobian ring of the Fermat hypersurface.

Lemma 7.4. Let $R$ be the Jacobian ring of the Fermat hypersurface of degree $d$ and dimension $n$. Let $p$ be an integer in the range $(d$, top/2) and let $q$ be such that $p+q=$ top. Let $I$ be an ideal of $R$ generated by monomials of degree $p$. Then $\operatorname{dim} I^{q} \geqslant \operatorname{dim} I^{p}$.

Proof. Let $x^{K}$ be a monomial of $S$. Then the map $x^{K} \mapsto K$ defines a one-to-one correspondence between the monomials of $S$ and the points of the semigroup $\mathscr{S}=\left(\mathbf{Z}^{+}\right)^{n+2}$ such that multiplication of monomials corresponds to addition of their multi-indices. Let $\mathscr{J}$ be the subsemigroup of $\mathscr{S}$ corresponding to monomials in $J$, and let $\mathscr{R}$ be the complement of $\mathscr{J}$, which we can view as defining a generating set of monomials for the quotient ring $R$. If $\mathscr{I}$ is the subset of $\mathscr{R}$ corresponding to monomials in $I$, then what we must prove is that 
where we use the natural grading (partition) of $\mathscr{S}$ into subsets $\mathscr{S}^{k}$ of multi-indices of total degree $k$. To establish (\#), let $\mathbf{R} \mathscr{S}$ be the real vector space spanned by $\mathscr{S}$, and let $\mathbf{R} \mathscr{A}$ be the subspace spanned by a subset $\mathscr{A}$ of $\mathscr{S}$. Let $\mathbf{w}$ be the vector orthogonal to $\mathbf{R} \mathscr{S}^{p}$ such that $\mathbf{R} \mathscr{S}^{q}=\mathbf{R} \mathscr{S}^{p}+\mathbf{w}$. Although the vectors of the set $\mathscr{I}^{p}+\mathbf{w}$ are not necessarily lattice vectors, one may approximate each element of $\mathscr{I}^{p}+\mathbf{w}$ by a closest lattice vector to obtain a subset $\mathscr{A}$ of $\mathscr{I}^{q}$ with the same cardinality as $\mathscr{I}^{p}+\mathbf{w}$, and thus with the same cardinality as $\mathscr{I}^{p}$. One verifies that if $x$ and $y$ are distinct elements of $\mathscr{I}^{p}+\mathbf{w}$, then their closest approximations in $\mathscr{I}^{p}$, no matter how chosen, are distinct as well: there is a small number $r$ such that for all $x$ the ball $B_{r}(x)$ of radius $r$ about $x$ contains a vector of $\mathscr{I}^{q}$, and this number can be chosen such that balls with distinct centers in $\mathscr{I}^{p}+\mathbf{w}$ are disjoint.

We now resume the proof of Lemma 7.2. The essential idea is to use a lower semicontinuity argument to show that the set of hypersurfaces for which the result holds is a nonvoid Zariski open set. To this end, let $E$ be the set consisting of pairs $(I, X)$, where $X$ is a hypersurface of degree $d$ and where $I$ is an ideal of the Jacobian ring of $X$ which is generated in degree $r$ by $k$ elements. One observes that $E$ is a bundle over $B$, the set of hypersurfaces of degree $d$, with fiber a Grassmannian. The set of pairs $(I, X)$ with $\operatorname{dim} I^{s} \leqslant k$ is then a closed subvariety of $E$ as is its projection into $B$. Because of Lemma 7.3, this subvariety cannot contain the point corresponding to the Fermat hypersurface, so it must be a proper subvariety, as required.

REMARK 7.5. The set of monomials $\mathscr{I}$ corresponding to a monomial ideal of the Jacobian ring for the Fermat hypersurface is the intersection with $\mathscr{R}$ of a union of translates $\mathscr{R}+\mathbf{w}$, where $\mathbf{w}$ ranges over a set of generators for $\mathscr{I}$. Because $\mathscr{I}$ is then a union of truncated cones, the graph of the Hilbert function of $I$ rises rapidly to meet that of $R$. Consequently the dimension of $I^{q}$ tends to be rather much larger than that of $I^{p}$, where $p, q$, and $I$ are as in the lemmas above.

\section{BIBLIOGRAPHY}

1. A. Ash, D. Mumford, M. Rapoport and Y. Tai, Smooth compactification of locally simmetric rarieties, Math. Sci. Press, Brookline, Mass., 1975.

2. W. L. Baily and A. Borel. Compactification of arithmetic quotients of hounded sinmetric domains. Ann. of Math. (2) 84 (1966), 442-528.

3. A. Borel, Compact Clifford-Klein forms of symmetric spaces, Topology 2 (1963), 111-122.

4. J. Carlson and P. Griffiths, Infinitesimal variations of Hodge structures and the global Torelli prohlem. Journées de Geometrie Algébrique d'Angers, Sijthoff and Noordhoff, 1980, pp. 51-76.

5. J. Carlson, M. Green, P. Griffiths and J. Harris, Infinitesimal variations of Hodge structure. Compositio Math. 50 (1983), 109-205.

6. R. Donagi, Generic Torelli for projective varieties, Compositio Math. 50 (1983). 325-353.

7. M. (ireen, The period map for hypersurface sections of high degree of an arhitrary rariety. Compositio Math. 55 (1984), 1j5-156..

8 . . Letter of September 1984.

9. P. Griffiths, Periods of integrals on algebraic manifolds. II. Amer. J. Math. 90 (1968), 568-626. $805-865$

10. . Periods of rational integrals. I, II, Ann. of Math. (2) 90 (1969), 460-495, 498-591.

11. A. Grothendieck, Hodge's conjecture is false for trivial reasons. Topology 8 (1969), 299-303.

12. K. Kii. The local Torelli theorem for varieties with divisible canonical class, Math. USSR-Izv. 12 (1978), 53-67. 
13. F. S. Macaulay, Some properties of enumeration in the theory of modular sistems. Proc. London Math. Soc. 26 (1927), 531-555.

14. C. Peters and J. Steenbrink, Infinitesimal variations of Hodge structure and the generic Torelli problem for projective hypersurfaces, Progress in Math., no. 30, Classification of Algebraic and Analytic Manifolds, Birkhauser, Boston, Mass., 1983, pp. 399-463.

15. H. V. Pittie, Letter of April 1984.

16. W. Schmid, Variations of Hodge structure: the singularities of the period mapping. Invent. Math. 22 (1973). 211-319.

Dipartment of Mathematics, University of Utah, Salt Lake City, Utah 84112 